\title{
Reconstruction of pulmonary artery with porcine small intestinal submucosa in a lamb surgical model: Viability and growth potential
}

\author{
Lorenzo Boni, MD, ${ }^{\mathrm{a}}$ Fariba Chalajour, MD, ${ }^{\mathrm{a}}$ Takashi Sasaki, MD, ${ }^{\mathrm{a}}$ Radhika Lal Snyder, BA, ${ }^{\mathrm{a}}$ \\ Walter D. Boyd, MD, ${ }^{\mathrm{b}}$ R. Kirk Riemer, PhD, ${ }^{\mathrm{a}}$ and V. Mohan Reddy, $\mathrm{MD}^{\mathrm{a}}$
}

\begin{abstract}
Objectives: This study investigated the time-dependent remodeling and growth potential of porcine small intestine submucosa as a biomaterial for the reconstruction of pulmonary arteries in a lamb model.
\end{abstract}

\begin{abstract}
Methods: Left pulmonary arteries were partially replaced with small intestine submucosal biomaterial in 6 lambs. Two animals each were humanely killed at 1,3, and 6 months. Computed tomographic angiography, macroscopic examination of the implanted patch, and microscopic analysis of tissue explants were performed.
\end{abstract}

\begin{abstract}
Results: All animals survived without complications. Patency and arborization of the pulmonary arteries were detected 6 months after implantation. There was no macroscopic narrowing or aneurysm formation in the patch area. The luminal appearance of the patch was similar to the intimal layer of the adjacent native pulmonary artery. Scanning electron microscopy showed that the luminal surface of the patch was covered by confluent cells. Immunohistochemical examination confirmed endothelialization of the luminal side of the patch in all of the explanted patches. The presence of smooth muscle cells in the medial layer was confirmed at all time points; however, expression of elastin, growth of the muscular layer, and complete degradation of patch material were detectable only after 6 months. The presence of c-Kit-positive cells suggests migration of multipotent cells into the patch, which may play a role in remodeling the small intestine submucosal biomaterial.
\end{abstract}

Conclusions: Our data confirmed that remodeling and growth potential of the small intestine submucosal biomaterial are time dependent. Additional experiments are required to investigate the stability of the patch material over a longer period. (J Thorac Cardiovasc Surg 2012;144:963-9)

\section{Supplemental material is available online.}

The reconstruction of defective or hypoplastic pulmonary artery (PA) is a common surgical procedure performed in pediatric patients with congenital cardiovascular disease. Augmentation of the PA is often implemented during the first year of life and requires synthetic or biologic patch materials. Although the outcomes of surgically repaired hypoplastic PAs have improved, ${ }^{1}$ frequent reoperations are unavoidable because commercially available graft materials cannot grow with the child's growing vessels. In

\footnotetext{
From the Division of Pediatric Cardiac Surgery, ${ }^{\text {a }}$ Department of Cardiothoracic Surgery, Stanford University, Stanford, Calif; and the Department of Cardiothoracic Surgery, ${ }^{\mathrm{b}}$ UC Davis Medical Center, Sacramento, Calif.

This work was partially supported by the Oak Foundation.

Disclosures: Authors have nothing to disclose with regard to commercial support.

Read at the 92nd Annual Meeting of The American Association for Thoracic Surgery, San Francisco, California, April 28-May 2, 2012.

L.B. and F.C. contributed equally to this work.

Received for publication April 2, 2012; revisions received June 30, 2012; accepted for publication July 12, 2012; available ahead of print Aug 22, 2012.

Address for reprints: Fariba Chalajour, MD, Falk Cardiovascular Research Center, 300 Pasteur Dr, Stanford, CA 94305-5407 (E-mail: fchalajour@gmail.com). $0022-5223 / \$ 36.00$

Copyright (c) 2012 by The American Association for Thoracic Surgery http://dx.doi.org/10.1016/j.jtcvs.2012.07.024
}

addition to having growth potential, ideal vascular patches should be readily available, easy to handle, and resistant to degeneration or infection. ${ }^{2,3}$ Recently, the application of extracellular matrix (ECM) has been gaining more attention as a possible biomaterial for cardiovascular repair. ${ }^{4}$ ECM biomaterials are mammalian decellularized tissues with preserved ECM components that may be harvested from various tissue sources. ${ }^{5,6}$ Small intestinal submucosa (SIS) is an ECM biomaterial that is widely used in clinical and animal studies. ${ }^{7-13}$ The biologic and mechanical characteristics of SIS biomaterials depend on the source of tissue, the age of the animal, and the preparation process. $^{14,15}$ It has been shown that the alteration of biologic and mechanical properties of the implanted SIS patch biomaterials depends on the new in vivo microenvironment. ${ }^{16,17}$ The structural integrity of SIS biomaterial is critical to the stability of implantation; however, activation of the host healing process is closely dependent on the degradation of ECM SIS. ${ }^{5}$ A balance between the rate of ECM degradation and host remodeling is therefore critical for a successful surgical outcome.

Application of SIS biomaterial for the reconstruction of congenital vascular defects has shown promising results with regard to the patency of repaired vessels in pediatric patients $^{8}$; however, remodeling and growth potential of the SIS patch remain the major concern. The goal of this study was to investigate time-dependent remodeling and 


\section{Abbreviations and Acronyms \\ $\mathrm{ECM}=$ extracellular matrix \\ PA = pulmonary artery \\ SIS = small intestinal submucosa \\ $\mathrm{SMA}=$ smooth muscle actin \\ $\mathrm{vWF}=$ von Willebrand factor}

growth potential of porcine SIS biomaterial for the reconstruction of PA in a lamb surgical model.

\section{MATERIALS AND METHODS \\ Animals}

Six Suffolk lambs, weighing approximately 10 to $20 \mathrm{~kg}$, were used for this study (provided by Center for Laboratory Animal Science, Davis, Calif). All animals received humane care in compliance with the Guide for the Care and Use of Laboratory Animals (www.nap.edu/catalog/5140.html).

\section{Surgical Procedures}

Animals fasted for 24 to 48 hours before surgery. All surgical procedures were performed with aseptic technique. Anesthesia was induced either with intravenously administered ketamine hydrochloride (INN ketamine) and diazepam or with isoflurane by mask. After intubation, the animals were mechanically ventilated and anesthesia was maintained with inhalation of $1 \%$ to $2.5 \%$ isoflurane. The animals were placed in the right lateral decubitus position, their wool was clipped, and a sterile preparation of the area was accomplished. A left lateral thoracotomy was performed. The pericardium was opened anteriorly to the phrenic nerve, and the left PA was isolated. Heparin was administered intravenously at $300 \mathrm{U} / \mathrm{kg}$. Vascular clamps were used to clamp the left main PA temporarily. Partial resection of the vessel was made, and the SIS patch $(5 \times 10 \mathrm{~mm})$ was placed with 6-0 polypropylene running sutures. After de-airing, clamps were removed. Bupivacaine hydrochloride (INN bupivacaine; $0.25 \%-0.5 \%$ ) was injected into the area around thoracotomy for postoperative analgesia before closure of the chest. The chest was closed in the standard fashion, with placement of a temporary chest tube that was removed a few minutes later, just before the animal extubation on the operating table. Aspirin ( $325 \mathrm{mg}$ ) was given orally once a day for 7 days postoperatively to prevent acute thrombosis on the surface of implanted materials.

After recovery, the animals were housed in free-walking pens until elective killing. The patch materials were harvested at 1-, 3-, and 6-month intervals after implantation. Two lambs from each group were made to fast for 24 hours. Each animal was then intubated and anesthetized with isoflurane and mechanically ventilated. The chest was reopened, and macroscopic investigation was done to assess the size and shape of the vessels. Left PA pressures proximal and distal to the patch were recorded by direct puncture. Finally, the animals were humanely killed with intravenous pentobarbital or intravenous saturated potassium chloride, and the vessels were harvested for histological study.

\section{Computed Tomographic Angiography}

Patency of the graft was assessed in all animals by macroscopic examination and direct pressure measurement at the moment of the explants. Furthermore, 2 animals underwent computed tomographic angiographic scan of the lungs at 6 months after implantation to assess patency and arborization pattern of the branch PAs before death.

\section{Scanning Electron Microscopy}

Small longitudinal sections of the explanted grafts were fixed for scanning electron microscopy (model S-3400N VP-SEM; Hitachi, Tokyo,
Japan). Scanning electron microscopy was performed to show the appearance of the luminal surface of the patched area.

\section{Histologic and Immunohistochemical Studies}

The explanted SIS vascular patch, along with a piece of native PA, was dissected and fixed in 10\% buffered formalin (Fisher Scientific, Fair Lawn, $\mathrm{NJ})$. The tissues were embedded in paraffin and, 5- $\mu \mathrm{m}$ tissue sections were prepared for Russell-Movat pentachrome staining (American Master Tech Scientific, Inc, Lodi, Calif). Immunohistologic analyses were performed for the endothelial cell marker von Willebrand factor (vWF; Dako Cytomation, Glostrup, Denmark), smooth muscle actin (SMA; Dako Cytomation), elastin (Abcam Inc, Cambridge, Mass), and the stem cell marker CD117 (c-Kit; Dako Cytomation). The thickness of the tissue explants was measured with image analysis software (LAS AF image analysis; Leica Microsystems Inc, Buffalo Grove, Ill).

\section{RESULTS}

\section{Postoperative Course and Gross Examination}

All 6 lambs survived surgery and the postoperative period without complications or signs of infection. The mean animal weight increased from $17.5 \mathrm{~kg}$ at the first operation to $23.9,51.7$, and $53.4 \mathrm{~kg}$ at 1,3 , and 6 months, respectively. At the time of explantation of patch material, the areas around the left PA and patch material presented a normal appearance. Formation of adhesion fibers around the suture lines was found 1 month after the first operation (Figure 1, $A$ ), and was reduced 6 months after implantation. The patched area did not show any narrowing or aneurysm formation, nor was there a pressure gradient across the patch. The luminal surface of the explanted patch was shiny and smooth, similar to the luminal surface of adjacent native left PA (Figure 1, $B$ ). This was observed at all time points. There was no thrombus formation on the endothelial surface of any explanted graft.

\section{In Vivo Imaging Analysis}

The computed tomographic angiographic scan with 3-dimensional reconstruction showed patency of the left PA without any sign of stenosis or aneurysm formation. The left and right PA sizes were normal and comparable with one another, as was the distal arborization after each PA branch (Figure 2).

\section{Scanning Electron Microscopy}

The scanning electron microscopy revealed the endoluminal aspect of the patch to be covered by confluent cells with the morphologic characteristics of endothelial cells from the first month after implantation (Figure 3).

\section{Histologic and Immunohistochemical Studies}

Our histologic analysis showed recellularization of grafted patch after 1 month (Figure 4, A); however, formation of ECM was limited to proteoglycan (Figure 4, $B$ ), in contrast to elastin fibers in native artery (Figure $4, C$ ). A diffuse infiltration of inflammatory cells into the adventitia increased the thickness of the grafted patch (Figure $4, D$ ) 


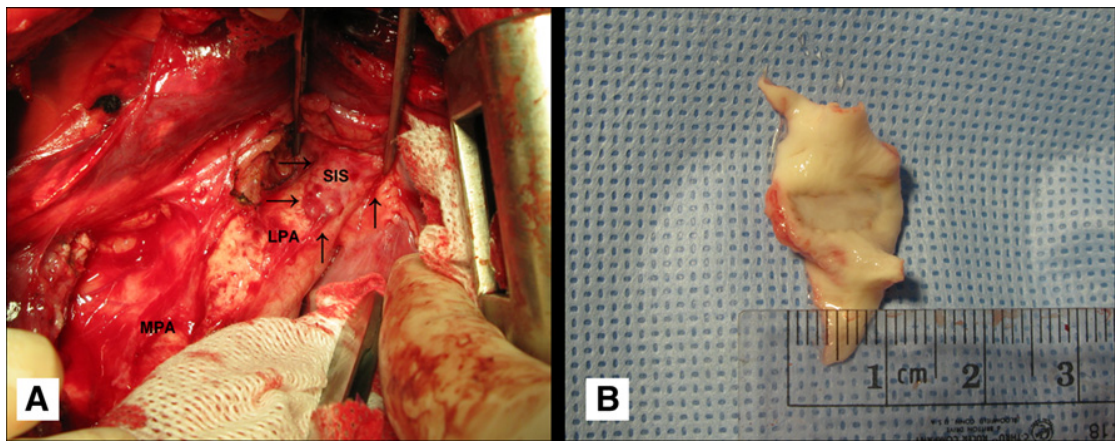

FIGURE 1. A, In situ macroscopic appearance of patched area 1 month after implantation. Arrows show patched area externally. There were no visible adherences around the patch area. The external caliber of the left pulmonary artery $(L P A)$ was free from stricture formation and distortions. B, Macroscopic view of the luminal surface of the explanted patch 1 month after implantation shows the patched area in the middle of the specimen (the left pulmonary artery was opened longitudinally). The intimal layer was found covering the luminal surface of the constructed left pulmonary artery, including the patched area. $M P A$, Main pulmonary artery; SIS, small intestine submucosal patch.

relative to the adventitia of native PA (Figure $4, E$ ). An increase in the size of the arterial lumen was observed 3 months after implantation (Figure $4, F$ ). The activation of the repair process within the patch was confirmed by the detection of newly formed blood vessels in the medial part of patch (Figure $4, G$ ) and also by the reduction of inflammatory response in the adventitia (Figure $4, I$ ). In addition, alteration of the ECM was observed by the production of collagen in the subintimal and medial parts of grafted patch. Six months after implantation, the morphology of the SIS patch mimicked the structure of native arterial wall (Figure $4, K$ ). The formation of elastin fibers provided evidence for arterialization of SIS patch (Figure 4, L).

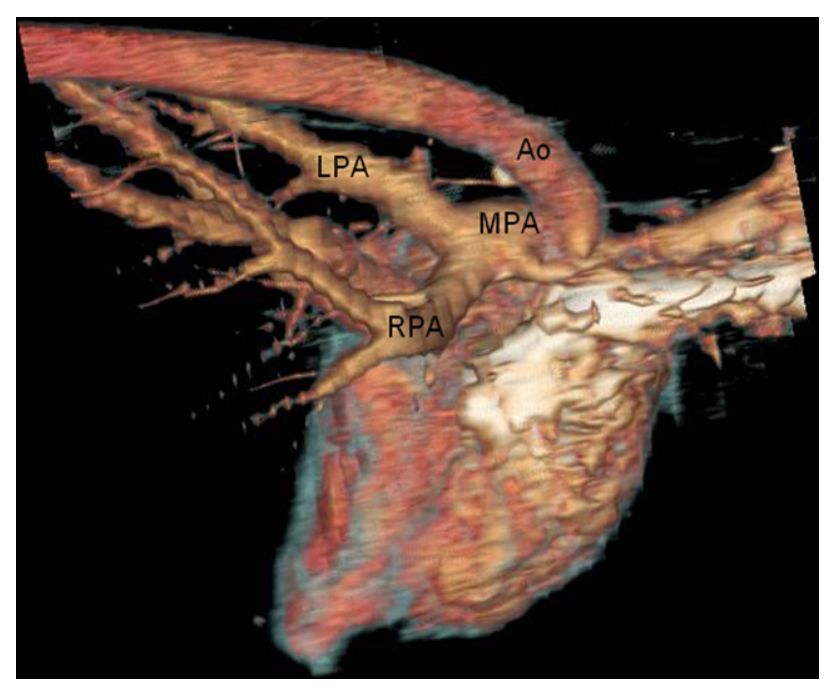

FIGURE 2. Computed tomographic angiographic scan with 3-dimensional reconstruction 6 months after patch implantation. The left pulmonary artery $(L P A)$ was patent, and there were no signs of stenosis or aneurysm formation. The left pulmonary artery and right pulmonary artery $(R P A)$ sizes were normal and comparable with each other, as was the distal arborization after each pulmonary arterial branch. Ao, Aorta; MPA, main pulmonary artery.
Furthermore, the reduction of inflammatory response and a consequent reduction of patch thickness were observed (Figure 4, $N$ ).

To characterize the cellular structure of implanted patch, immunohistologic analysis was performed. Endothelialization of the luminal side of the SIS patch was confirmed by the expression of endothelial cell marker vWF in the cells covering the lumen 1,3 , and 6 months after implantation (Figure 5, $A, E$, and $I$, respectively). The presence of smooth muscle cells in the medial layer of the patch was confirmed by the expression of SMA after 1 month (Figure 5, B), 3 months (Figure 5, F), and 6 months (Figure 5, $J$ ). The expression of elastin fibers required a longer period, however, and was detectable only in the 6-month vascular patch (Figure 5, $K$ ). The thickness of the tunica media in the native left PA was $2400 \mu \mathrm{m}$, and the muscle bundle was around $1200 \mu \mathrm{m}$ (almost half the arterial wall thickness). The

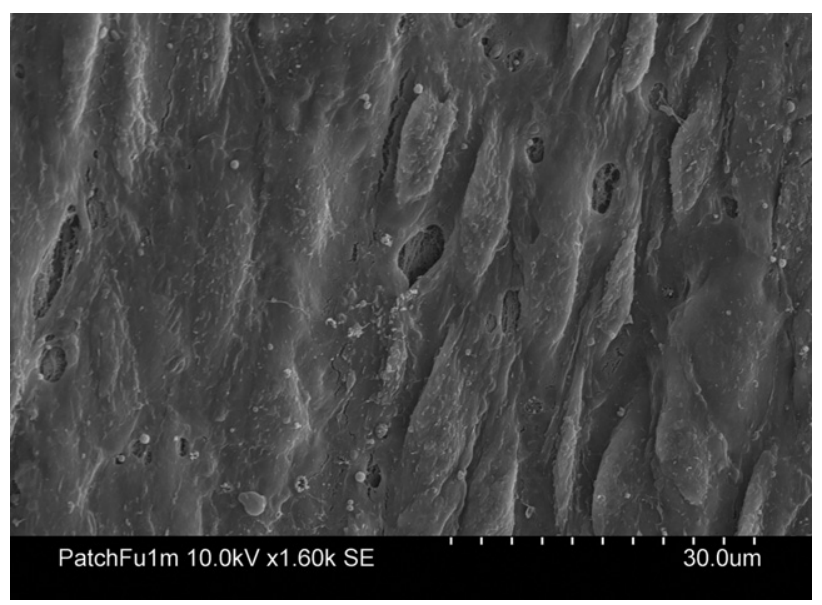

FIGURE 3. Scanning electron microscopy of the luminal surface of the small intestine submucosal patch 1 month after implantation. The patch area was covered with confluent cells with the morphologic characteristics of endothelial cells. Original magnification $\times 1600$. 


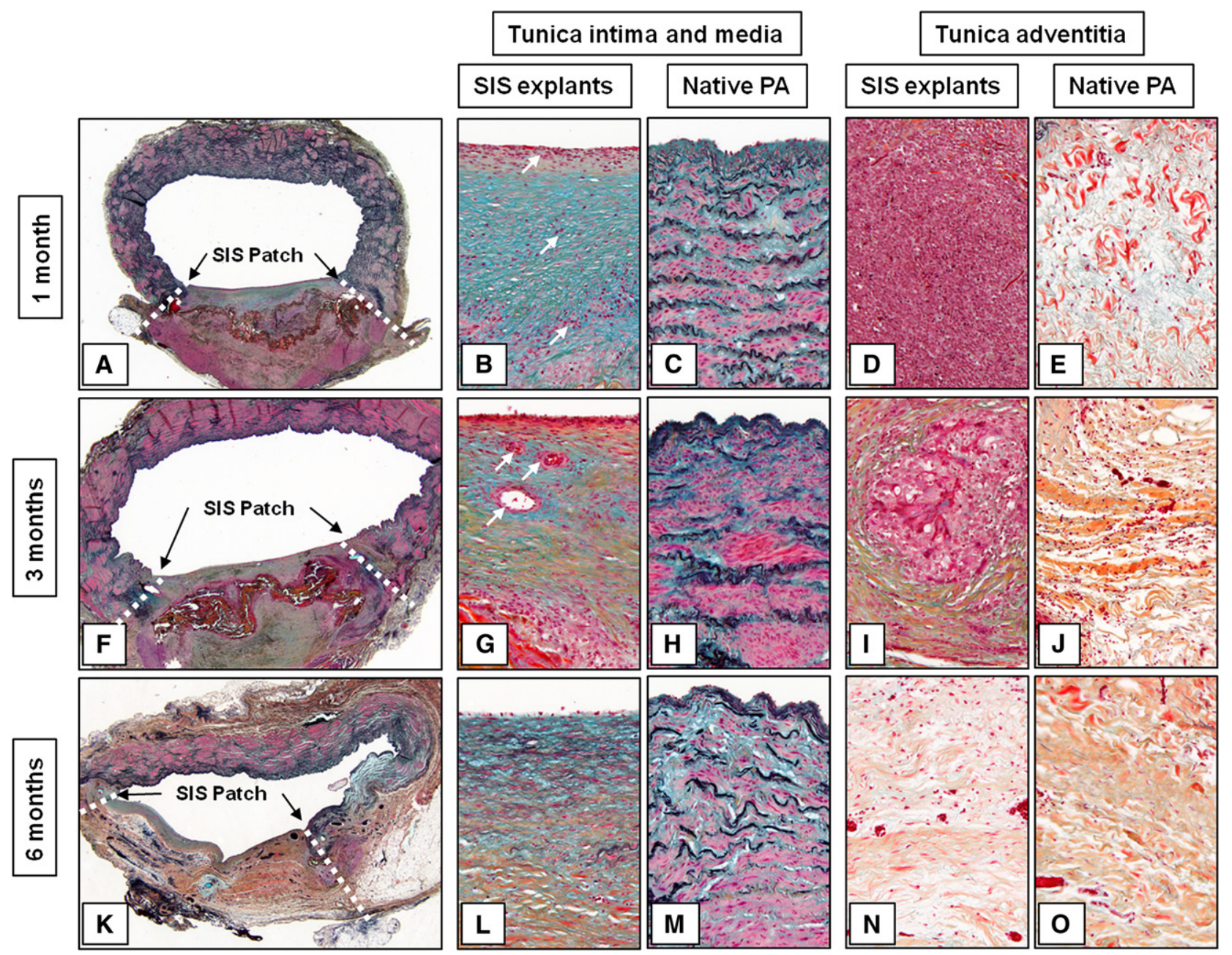

FIGURE 4. Russell-Movat pentachrome staining of small intestine submucosal (SIS) patch explants. The cross sections of small intestine submucosal patch tissue explants after 1 month (A) shows cellularization of intima and media (arrows; B). Formation of extracellular matrix is confirmed by production of collagen (yellow) in subintimal layer and proteoglycans (blue) in the tunica media; however, elastin fibers (black) are only detected in the wall of native pulmonary artery (C), not in the small intestine submucosal patch. Comparison of tunica adventitia in the small intestine submucosal explants (D) with native pulmonary artery (E) shows migration of inflammatory cells into the patch, thus increasing the thickness of patch. The cross section of small intestine submucosal patch tissue explants after 3 months shows increasing the size of pulmonary arterial lumen $(\mathrm{F})$ and formation of blood vessels in the tunica media (arrows; G). Partial replacement of proteoglycans by collagen is evidence that matrix production in the small intestine submucosal patch tunica media is dynamic. Distribution of proteoglycans in small intestine submucosal patch follows a pattern similar to that in native pulmonary artery (H). No elastin fiber is observed in the small intestine submucosal patch after 3 months. Although the number of inflammatory cells in the tunica adventitia of the 3-month-old small intestine submucosal patch (I) is higher than that in the native pulmonary artery (J), it is dramatically decreased relative to a 1-month sample (D). The cross section of small intestine submucosal patch tissue explants after 6 months shows reduction of patch thickness (K) and formation of elastin fiber in the patch (L) with a pattern similar to that in native pulmonary artery (M). The similarity in the distribution of cells and extracellular matrix between small intestine submucosal patch tunica adventitia $(\mathrm{N})$ and native pulmonary artery $(\mathrm{O})$ indicates the reduction of inflammatory response after 6 months. Original magnifications $\times 16(\mathrm{~A}, \mathrm{~F}$, and $\mathrm{K})$ and $\times 400(\mathrm{~B}-\mathrm{E}, \mathrm{G}-\mathrm{J}$, and L-O).

thickness of newly formed muscle layer in the patch was $350 \mu \mathrm{m}$, and it was more than a fifth of the $1750-\mu \mathrm{m}$ thickness of the whole patch.

The existence of the stem cell marker CD117 within the grafted patch after 1, 3, and 6 months (Figure 5, D, H, and $L$, respectively) suggested a source of multipotent cells that can differentiate into the required vascular cells for repopulation of the SIS patch. Although we could not show the origin of $\mathrm{CD} 117^{+}$cells, the age-dependent increase in the number of $\mathrm{CD} 117^{+}$cells suggested that these cells may contribute to the growth and remodeling of the SIS patch.

Vascularization of the SIS patch 1 and 3 months after implantation (Figure E1, $A$ and $B$, respectively) provides additional evidence for the activation of the proliferative phase of the remodeling process. Regression of newly formed 


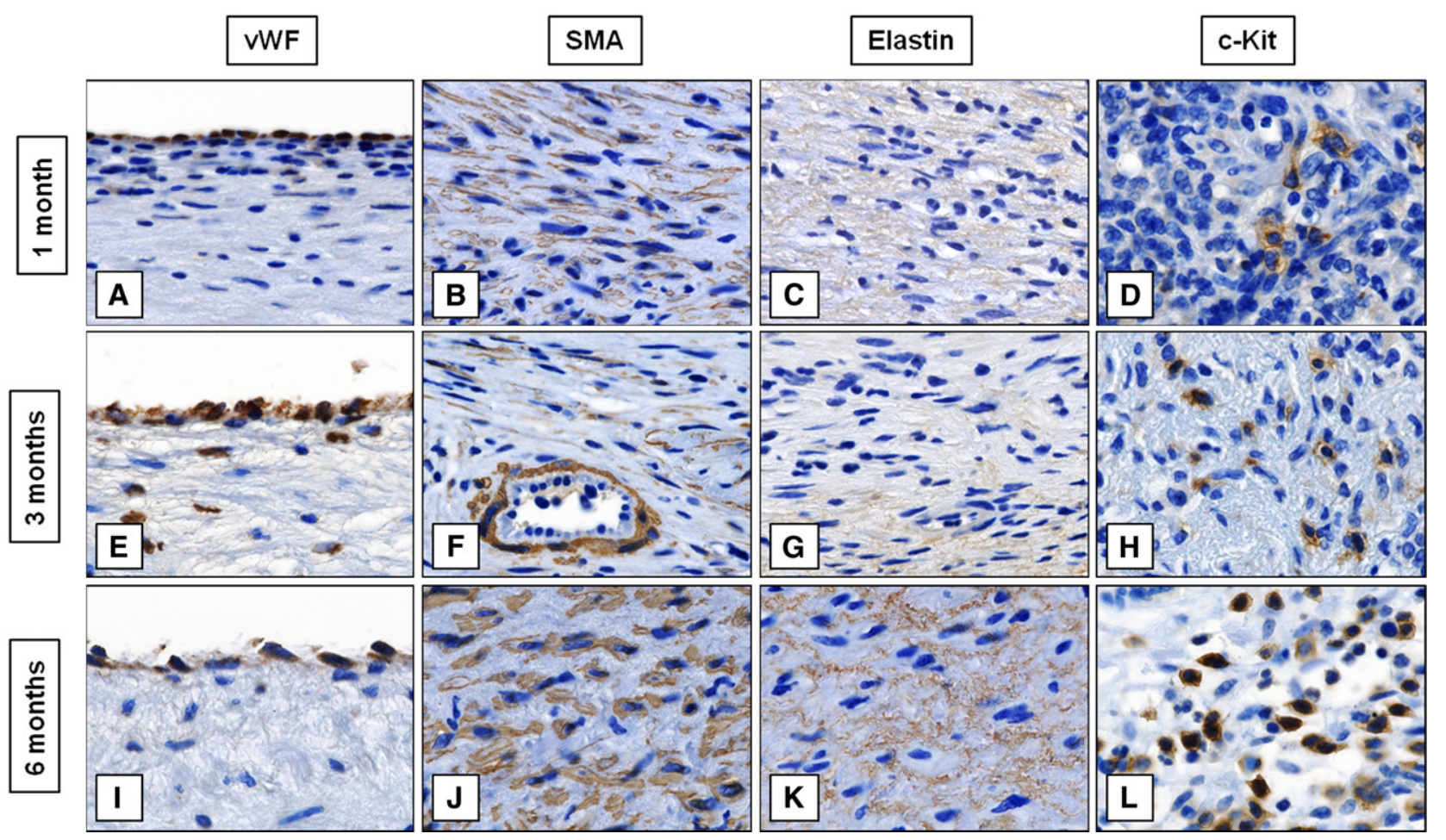

FIGURE 5. Immunohistologic analysis of porcine small intestine submucosal patch explants. Formation of tunica intima was confirmed by expression of endothelial cell marker von Willebrand factor $(v W F)$ in the cells covering the luminal side of grafted patch 1 month, 3 months, and 6 months after implantation (A, E, and I, respectively). Expression of smooth muscle actin (SMA) was observed in the medial layer of small intestine submucosal patch after 1 month (B), 3 months (F), and 6 months (J); however, the expression of elastin was observed in the medial layer of only 6-month small intestine submucosal patch $(\mathrm{K})$, not in the 1-month $(\mathrm{C})$ and 3-month $(\mathrm{G})$ samples. The expression of stem cell marker CD117 (c-Kit) was identified in the small intestine submucosal patch 1 month (D), 3 months (H), and 6 months (L) after implantation; however, the number and distribution of CD117 cells increased with time, indicating the remodeling potential of small intestine submucosal patch. Original magnification $\times 1000$ (A-L).

tissue suggests maturation of the remodeling process after 6 months (Figure 1,C), at which time the perfused vessels were mostly localized in the adventitia to supply the new arterial wall.

\section{DISCUSSION}

In this study, we investigated time-dependent recellularization and remodeling of porcine SIS grafted into the left PA in a lamb model. The vascular structure of the SIS graft was determined by histologic and immunohistologic staining at 1-, 3-, and 6-month intervals after implantation. Formation of tunica intima was confirmed by expression of vWF on the luminal side of the SIS patch in all 3 groups. Expression of SMA in the medial layer of the SIS patch indicated the formation of tunica media; however, the formation of elastic fibers was evident later and was not detectable until 6 months after implantation. The wound healing process was initiated by the recruitment of inflammatory cells and was continued by the vascularization of SIS patch 1 month and 3 months after implantation, respectively. Resolution of inflammation and regression of newly formed blood vessels within the patch were evident 6 months after implantation. The patency of the reconstructed PA was confirmed by direct measurement of arterial pressure, computed tomographic angiographic scan, and macroscopic examination at explantation. The increasing thickness of the implanted SIS patches was measured microscopically. Identification of $\mathrm{CD}_{117^{+}}$cells within the patch suggests the possible contribution of hematopoietic stem cells to the remodeling of the SIS patch.

The application of autograft SIS biomaterial for reconstruction of superior and inferior venae cavae was introduced by Matsumoto and colleagues ${ }^{18}$ in 1966. Since then, many efforts have been made to improve the biologic and mechanical properties of SIS biomaterials. The ECM components provide a suitable matrix for the migration of inflammatory cells, circulating bone marrow-derived cells, and cells from the adjacent native tissue into the ECM matrix. ${ }^{19,20}$ The differentiation of the migrated cells into endothelial and smooth muscle cells is critical to recellularization of the vascular patch biomaterial. Endothelialization of the SIS vascular patch was detectable 1, 3, and 6 months after implantation. The presence of smooth muscle cells was confirmed by expression of SMA in the cells located in the medial layer of the patch. Production of elastic fibers has been 
identified as characteristic of arterial smooth muscle cells. ${ }^{21}$ Production of elastic fibers was time dependent, however, and it was only detectable 6 months after implantation of the SIS patch. Elastin plays an important role in the vascular structure and function in response to mechanical force. The critical role of elastic fibers in the stability of the arterial wall has been studied in Marfan syndrome, in which the degradation of elastic fibers in the large arteries results in aneurysms. ${ }^{22}$ Although we did not find aneurysms in the implanted vascular patches, the slow development of elastic fibers in the SIS vascular patch and subsequent potential formation of aneurysms remains a possible concern during a longer period, as reported by other investigators. ${ }^{23}$

Activation of inflammatory and wound healing processes during the remodeling of different kinds of biomaterials has been reported by other investigators. ${ }^{24,25}$ It has been shown that macrophages and lymphocytes play pivotal roles in the initiation of the matrix remodeling. ${ }^{19}$ In this study, infiltration of mononuclear cells was detectable in all the explanted SIS patches; however, the number of infiltrated cells decreased massively 6 months after implantation. Activation of angiogenesis and deposition of collagen characterized the proliferative phase of the SIS patch remodeling 3 months after implantation. The regression of newly formed vessels in the medial layer and the localization of small vessels in the adventitial layer indicated formation of vasa vasorum externa in the patch 6 months after implantation.

The rate of in vivo degradation of SIS biomaterial is critical for the initiation of the healing process ${ }^{5}$; however, rapid absorption of the ECM biomaterial decreases the stability of the implanted patch and may cause rupture of the constructed vessel. The studies with carbon 14-labeled SIS showed degradation of $60 \%$ of the patch material during the first month and complete absorption of the patch material 3 months after repair of the urinary bladder in canine model. ${ }^{26}$ In our study, the patch material could be detected even 3 months after implantation. We speculate that the rate of patch degradation may depend on the type of recipient tissue and species.

The growing capacity of SIS biomaterial has been studied by Robotin-Johnson and coworkers. ${ }^{10}$ They used autologous SIS patches for reconstruction of piglet superior venae cavae. They reported increases in the circumference and length of implanted patches 3 months after implantation. ${ }^{10}$ In our study, microscopic measurements were used to evaluate the thickness of explanted patch material. Alteration of the patch thickness was correlated with the phase of patch remodeling. The thickness of the patch increased during the inflammatory and proliferative phases and then decreased 6 months after implantation. Despite the reduction in the patch thickness, the thickness of the tunica media increased 6 months after implantation. This suggests that development and organization of the muscular bundles might require a longer period than 6 months to reach the same thickness as native PA.

Our results showed patency, remodeling, and growth potential of the porcine SIS biomaterial for reconstruction of PAs in the lamb. More experiments with a greater number of animals from different species need to be performed to confirm the significance of these results. Long-term follow up is required to answer questions regarding stability and complete remodeling of vascular patches.

We acknowledge the excellent technical assistance of Xiaoyuan Ma and William T. Ferrier. We also thank CorMatrix ECM Technology, Alpharetta, Ga, for providing the porcine small intestine submucosal patches.

\section{References}

1. Reddy VM, Liddicoat JR, Hanley FL. Midline one-stage complete unifocalization and repair of pulmonary atresia with ventricular septal defect and major aortopulmonary collaterals. J Thorac Cardiovasc Surg. 1995;109:832-45.

2. Tang GH, Fazel S, Weisel RD, Van Arsdell GS, Li RK. Cardiovascular tissue engineering therapy: so near, so far? Ann Thorac Surg. 2005;79:1831-3.

3. Iwai S, Sawa Y, Taketani S, Torikai K, Hirakawa K, Matsuda H. Novel tissueengineered biodegradable material for reconstruction of vascular wall. Ann Thorac Surg. 2005;80:1821-7.

4. Piterina AV, Cloonan AJ, Meaney CL, Davis LM, Callanan A, Walsh MT, et al. ECM-based materials in cardiovascular applications: Inherent healing potential and augmentation of native regenerative processes. Int J Mol Sci. 2009;10: 4375-417.

5. Badylak SF, Freytes DO, Gilbert TW. Extracellular matrix as a biological scaffold material: Structure and function. Acta Biomater. 2009;5:1-13.

6. Cornwell KG, Landsman A, James KS. Extracellular matrix biomaterials for soft tissue repair. Clin Podiatr Med Surg. 2009;26:507-23.

7. Ueno T, Pickett LC, de la Fuente SG, Lawson DC, Pappas TN. Clinical application of porcine small intestinal submucosa in the management of infected or potentially contaminated abdominal defects. J Gastrointest Surg. 2004;8:109-12.

8. Quarti A, Nardone S, Colaneri M, Santoro G, Pozzi M. Preliminary experience in the use of an extracellular matrix to repair congenital heart diseases. Interact Cardiovasc Thorac Surg. 2011;13:569-72.

9. Lantz GC, Badylak SF, Hiles MC, Coffey AC, Geddes LA, Kokini K, et al. Small intestinal submucosa as a vascular graft: a review. J Invest Surg. 1993;6: 297-310.

10. Robotin-Johnson MC, Swanson PE, Johnson DC, Schuessler RB, Cox JL. An experimental model of small intestinal submucosa as a growing vascular graft. $J$ Thorac Cardiovasc Surg. 1998;116:805-11.

11. Fallon A, Goodchild T, Wang R, Matheny RG. Remodeling of extracellular matrix patch used for carotid artery repair. J Surg Res. 2012;175:e25-34.

12. Du XF, Kwon SK, Song JJ, Cho CG, Park SW. Tracheal reconstruction by mesenchymal stem cells with small intestine submucosa in rabbits. Int J Pediatr Otorhinolaryngol. 2012;76:345-51.

13. Ruiz CE, Iemura M, Medie S, Varga P, Van Alstine WG, Mack S, et al. Transcatheter placement of a low-profile biodegradable pulmonary valve made of small intestinal submucosa: a long-term study in a swine model. $J$ Thorac Cardiovasc Surg. 2005;130:477-84.

14. Tottey S, Johnson SA, Crapo PM, Reing JE, Zhang L, Jiang H, et al. The effect of source animal age upon extracellular matrix scaffold properties. Biomaterials. 2011;32:128-36.

15. Deeken CR, Eliason BJ, Pichert MD, Grant SA, Frisella MM, Matthews BD. Differentiation of biologic scaffold materials through physicomechanical, thermal, and enzymatic degradation techniques. Ann Surg. 2012;255:595-604.

16. Hiles MC, Badylak SF, Lantz GC, Kokini K, Geddes LA, Morff RJ. Mechanical properties of xenogeneic small-intestinal submucosa when used as an aortic graft in the dog. J Biomed Mater Res. 1995;29:883-91.

17. Badylak SF, Kropp B, McPherson T, Liang H, Snyder PW. Small intestinal submucosa: a rapidly resorbed bioscaffold for augmentation cystoplasty in a dog model. Tissue Eng. 1998;4:379-87.

18. Matsumoto T, Holmes RH, Burdick CO, Heisterkamp CA 3rd, O'Connell TJ Jr. Replacement of large veins with free inverted segments of small bowel: 
autografts of submucosal membrane in dogs and clinical use. Ann Surg. 1966; 164:845-8.

19. Badylak SF, Gilbert TW. Immune response to biologic scaffold materials. Semin Immunol. 2008;20:109-16.

20. Badylak SF, Park K, Peppas N, McCabe G, Yoder M. Marrow-derived cells populate scaffolds composed of xenogeneic extracellular matrix. Exp Hematol. 2001;29:1310-8.

21. Narayanan AS, Sandberg LB, Ross R, Layman DL. The smooth muscle cell. III. Elastin synthesis in arterial smooth muscle cell culture. J Cell Biol. 1976;68: 411-9.

22. Chung AW, Au Yeung K, Sandor GG, Judge DP, Dietz HC, van Breemen C. Loss of elastic fiber integrity and reduction of vascular smooth muscle contraction resulting from the upregulated activities of matrix metalloproteinase-2 and -9 in the thoracic aortic aneurysm in Marfan syndrome. Circ Res. 2007; 101:512-22.

23. McCready RA, Hodde J, Irwin RJ, Coffey AC, Divelbiss JL, Bryant MA, et al. Pseudoaneurysm formation in a subset of patients with small intestinal submucosa biologic patches after carotid endarterectomy. J Vasc Surg. 2005;41: 782-8.

24. Ziats NP, Miller KM, Anderson JM. In vitro and in vivo interactions of cells with biomaterials. Biomaterials. 1988;9:5-13.

25. Anderson JM, Rodriguez A, Chang DT. Foreign body reaction to biomaterials. Semin Immunol. 2008;20:86-100.

26. Record RD, Hillegonds D, Simmons C, Tullius R, Rickey FA, Elmore D, et al. In vivo degradation of $14 \mathrm{C}$-labeled small intestinal submucosa (SIS) when used for urinary bladder repair. Biomaterials. 2001;22:2653-9.

\section{Discussion}

Dr Frank A. Pigula (Boston, Mass). Dr Boni, thank you for a good presentation and a good manuscript, and congratulations to your team with this model. These are all difficult models to perform.

I would note that this material, this SIS, has been used for vascular reconstructions previously. And in fact, this particular preparation has been approved by the Food and Drug Administration for 3 applications: for pericardial replacement, for intracardiac patching, and, more recently, in fact late last year, for carotid artery patching as well.

With that being said, this is among the more careful studies that I have come across in the literature. You have shown to my satisfaction that there is a process of change in this SIS graft after implantation and that it appears to transform into PA-like tissue with time. I do, however, have a couple questions.

One is that you suggested that there was growth potential in the tissue. Now, did you measure the patch that you put in or measure the patch that you took out? And how can you be sure that it was not compensatory growth from the surrounding PA?

Dr Boni. Thank you for the question, Dr Pigula.

We did measure the patch. We found that the patch, in the first moment, was increasing its thickness, and probably this was due to the activation of the inflammatory process, which we could see mainly in the adventitial layer, where we saw a lot of inflammatory cells that populated the patch. The reason that we suggest the growing idea is that when we later assessed the thickness of the patch, we saw that the medial layer was increasing or growing, but the full thickness of the patch was decreasing. So the inflammation started to regress after 3 months, and the medial layer started increasing in size. And apart from this, we could see the appearance of elastin fibers, which is a marker of an arterial wall.

We cannot say for sure that the patch was growing. We do know that it was getting repopulated by cells, and we do not know exactly from where they came. We saw $\mathrm{CD} 117^{+}$cells, but we do not know how it was going, the processing.

Dr Pigula. I guess when I was referring to growth, I was referring to the surface area growth, rather than the thickness.

Dr Boni. Yes, that's why I think we probably need longer experiments, because I think the PA wall is an elastic tissue, and we may need to place bigger patches and follow up for much longer to see that it is growing in this area.

Dr Pigula. Well, that brings me to my other question regarding the thickness of the patch and the composition of the patch with time. You have shown that there was elastin in the tissue at the end of the 6 months, but it was a fraction of what there is in the normal PA. So one of the questions for people who may be implanting this is what is the mechanical strength of this tissue? And if there is a transformative process, it is a balance between degradation of one and regeneration of another and having a mechanically safe tissue.

Dr Boni. Yes, of course, this is a very good point. We did not measure the mechanical force. Other studies did it before and demonstrated a good mechanical force.

And also, measuring the patch, we saw that, for example, relative to the native PA, the medial layer was more or less a fifth the full thickness in the patch, whereas in the arterial wall it was half, with elastin fibers, a few fibers, at 6 months. So that is why I say that we started to see that the patch was mimicking the arterial wall, because probably this process was still ongoing. And we should follow up such animals and do other studies for a longer period to assess the complete remodeling, and probably to assess the mechanical force.

We did not see any aneurysm formation, so in this study we could not see any problem with the weakness of the patch.

Dr Pigula. Is it being used clinically in your institution?

Dr Boni. Yes, we have been using it clinically. In tiny PAs, when they are very small, smaller than $3 \mathrm{~mm}$, we have had some problem, probably because of the initial phase of remodeling, when the inflammatory process is higher, and we have had some stenosis of these small structures. So we use it in bigger structures, and also in PAs but not the tiny ones. 

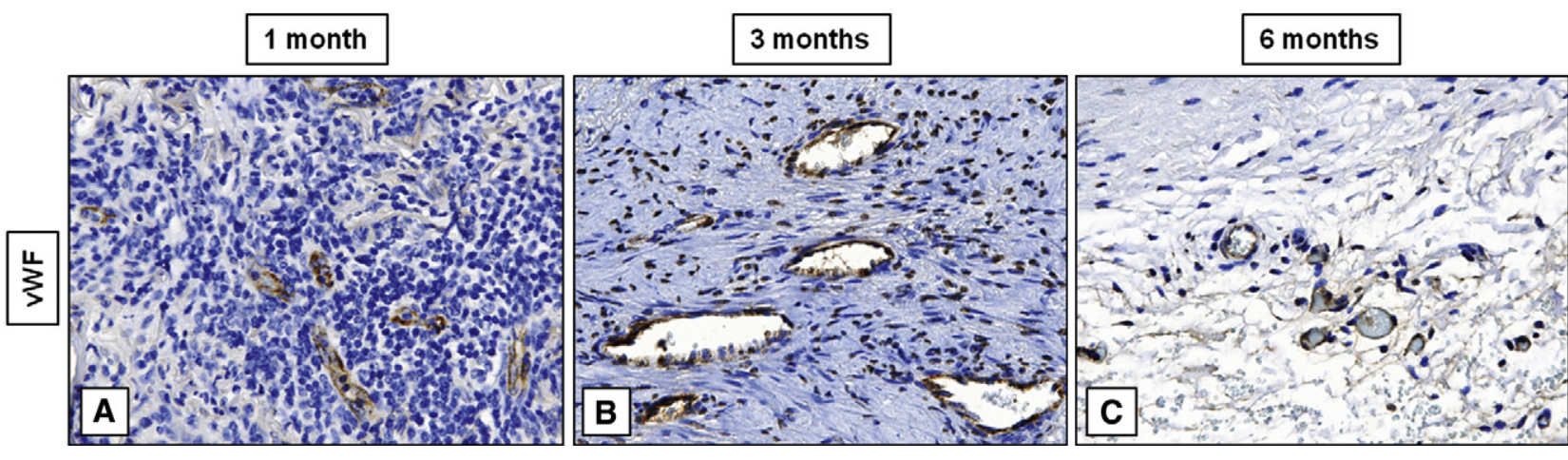

FIGURE E1. Activation of angiogenesis in small intestine submucosal patch. Vascularization of small intestine submucosal patch was confirmed by expression of endothelial marker von Willebrand factor $(v W F)$ in the lumen of newly formed blood vessels in 1-month (A), 3-month (B), and 6-month (C) explants. The highest density of blood vessels was observed 3 months after patch implantation (B), demonstrating the proliferative phase of patch repair, whereas regression of blood vessels in the medial layer was identified in 6-month samples (C), indicating maturation and remodeling of grafted patch. Original magnification $\times 400($ A-C). 